\title{
Allopregnanolone concentrations and premenstrual syndrome
}

Patrizia Monteleone, Stefano Luisi, Arianna Tonetti, Francesca Bernardi, Alessandro D Genazzani² , Michele Luisi ${ }^{1}$, Felice Petraglia ${ }^{3}$ and Andrea R Genazzani

Department of Reproductive Medicine and Child Development, Division of Gynaecology and Obstetrics 'P. Fioretti', University of Pisa, ${ }^{1}$ CNR, Endocrine Research Unit, Pisa, ${ }^{2}$ Institute of Obstetrics and Gynecology, University of Modena, Modena and ${ }^{3}$ Department of Surgical Sciences, Division of Gynecology and Obstetrics, University of Udine, Udine, Italy

(Correspondence should be addressed to A R Genazzani, Department of Reproductive Medicine and Child Development, Division of Gynaecology and Obstetrics 'P. Fioretti', University of Pisa, Via Roma 35, 56100 Pisa, Italy; Email: a.genazzani@obgyn.med.unipi.it)

\begin{abstract}
Objective: To evaluate basal allopregnanolone and progesterone in both phases of the menstrual cycle in women suffering from premenstrual syndrome (PMS) and their response to a GnRH test. Design: We selected 56 women (28 patients with PMS and 28 controls) aged between 18 and 32 years. Blood samples were drawn in both follicular and phases. Twenty-eight women (14 patients with PMS and 14 controls) underwent a GnRH test in the luteal phase.

Methods: We evaluated allopregnanolone by RIA, using a specific antibody. Serum progesterone and oestradiol were determined using a commercially available RIA kit.

Results: Luteal phase allopregnanolone concentrations were significantly lower in patients with PMS than in controls. Progesterone concentrations were significantly lower in patients with PMS in both the follicular and the luteal phase. Serum oestradiol concentrations were in the normal range in both groups of women, although slightly greater in those with PMS. Allopregnanolone and progesterone responses to a GnRH test were significantly blunted in women with PMS.

Conclusions: Diminished concentrations of allopregnanolone and progesterone, its precursor, and a blunted response to the GnRH test lead us to hypothesise that patients with PMS may suffer from an inadequate production of ovarian neuroactive steroids, especially in the luteal phase. This would lead to an impaired anxiolytic $\mathrm{GABA}_{\mathrm{A}}$-mediated response in stressful physiological and psychological conditions, and may in part explain various psychoneuroendocrine symptoms that arise during PMS.
\end{abstract}

European Journal of Endocrinology 142 269-273

\section{Introduction}

Premenstrual syndrome (PMS) is characterised by the cyclic appearance of distressing behavioural and somatic symptoms during the luteal phase, followed by a period of time entirely free of symptoms. Although there is no proof as to what really causes PMS, factors produced by the corpus luteum may be involved in eliciting this disorder: PMS begins on corpus luteum formation and disappears with luteolysis. Investigators have attempted to identify a deficiency or excess of a luteal phase-related factor, active within the central nervous system, that could mediate premenstrual anxiety symptoms. Despite observations of the manifold neuroregulatory effects of both oestrogen and progesterone, no consistent abnormality of these hormones has been demonstrated $(1,2)$. Recent studies report that they may have a role in determining PMS $(3,4)$, whereas data regarding progesterone concentrations are quite conflicting.

Lately, one of the progesterone metabolites, allopregnanolone, has raised interest in various research groups. This neurosteroid is a 3- $\alpha, 5-\alpha$ reduced metabolite of progesterone. In common with other neuroactive steroids, it can interact with classical intracellular steroid receptors and bind to membrane receptors, therefore acting through both genomic and non-genomic mechanisms. Allopregnanolone influences the excitability of neurones by interacting with $\gamma$-aminobutyric acid $(\mathrm{GABA})_{\mathrm{A}}$ receptors $(5,6)$, and this may explain how it influences mood, behaviour, response to stress and cognitive functions. In the past, various authors have tried to find some correlation between psychoneuroendocrine symptoms characteristic of PMS and altered allopregnanolone concentrations (7-11). In normal women, allopregnanolone varies very similarly to progesterone throughout the menstrual cycle: they are both present in much greater concentrations in the luteal phase than in the follicular phase (12).

We evaluated basal allopregnanolone and progesterone, its precursor, and oestradiol in both the follicular 
and luteal phases of the menstrual cycle over 3 months in patients with prospectively confirmed PMS and in asymptomatic controls. We also performed a gonadotrophin-releasing hormone (GnRH) test during the luteal phase of the third month, in order to verify any difference in ovarian allopregnanolone response between the two study groups.

\section{Participants, materials and methods}

Among all patients referred to our Department, a group of 28 women, from a total of 110 seeking help for PMS (25.5\%), were selected (age range 18-32 years, mean 28 years). A group of 28 age-matched controls were selected among female students, fellows and doctors of our Department. All those included in the study had no evidence of psychiatric disease, assessed using DSM-IV criteria and none had other current or recent medical illness or had taken psychoactive medications, hormonal preparations (including oral contraceptives), mineral or vitamin supplements for at least 6 months before the study. All women with PMS subjects reported a consistent interference of their premenstrual disorder in their working, family or social relationships, and for all of them this PMS status was considered to affect all their activities significantly. They were all asked to keep a visual analogue scale diary to monitor their symptoms for the following three menstrual cycles, in order to chart accurately the cyclic nature of the symptoms. All patients had a history taken and a physical examination performed; body mass index varied within a $10 \%$ range with respect to ideal body weight. Ovulation was assessed by performing a luteinizing hormone assay. Each patient signed a consent form and the local Ethics Committee approved the study.

Blood sampling, for basal measurements, took place on three consecutive mornings between days 5-8 of the follicular phase and between days 22-26 of the luteal phase over 3 months, after overnight fasting. In 14 patients with PMS aged 18-32 years (mean 26 years) and 14 age-matched controls, we performed an acute GnRH stimulation test in the luteal phase of the third month; blood samples were collected at baseline $(t=-15 \mathrm{~min}, 0)$ and every $15 \mathrm{~min}$ for $2 \mathrm{~h}$ after i.v. bolus injection of $100 \mu \mathrm{g}$ GnRH (GnRH, Serono, Milan, Italy). Blood was centrifuged and serum samples were then stored at $-20^{\circ} \mathrm{C}$ until required for assay.

Allopregnanolone assay was performed after ether extraction and chromatographic partition on C18 SepPak cartridges by using a previously described method (12). Polyclonal antiserum, raised in sheep injected with allopregnanolone carboxylmethylether coupled to BSA, was kindly provided by Dr RH Purdy (San Diego, CA, USA). The sensitivity of the assay, expressed as the minimal amount of allopregnanolone distinguishable from the zero sample with $95 \%$ probability, was $15-$ $20 \mathrm{pg} /$ tube and the intra- and interassay coefficients of variation were $7.2 \%$ and $9.1 \%$, respectively. Serum progesterone and oestradiol were determined, after ether extraction and chromatographic partition on C18 Sep-Pak cartridges, using a commercially available RIA kit (Radim SpA, Pomezia, Italy). The sensitivity for the progesterone assay was $30 \mathrm{pg} /$ tube, with intraand interassay coefficients of variation being $6.5 \%$ and $8.7 \%$, respectively; for oestradiol, sensitivity was $10 \mathrm{pg} /$ tube and the intra- and interassay coefficients of variation were $2.4 \%$ and $3.5 \%$, respectively.

\section{Statistical analysis}

The presence of significant difference between groups was tested, after analysis of variance (one-way ANOVA), using Student's $t$-test for paired and unpaired data, as appropriate. Results are expressed as mean \pm S.E.M.

\section{Results}

Mean baseline serum allopregnanolone, progesterone and oestradiol concentrations over 3 months are reported in Table 1 . There was no significant difference between the groups with respect to duration of the menstrual cycle (PMS: $33 \pm 1.2$ days, controls: $32 \pm$ 1.0 days) and basal values of allopregnanolone, progesterone and oestradiol over 3 months. Progesterone concentrations were significantly lower in patients with PMS than in controls in both the follicular and the luteal phase $(P<0.0001)$. Allopregnanolone concentrations were significantly lower in the serum of patients suffering from PMS $(P<0.0001)$ only in the luteal phase. Moreover, the ratio between luteal phase and follicular phase allopregnanolone concentrations in our patients with PMS was three times less than that of symptom-free women. There was no positive correlation between allopregnanolone and progesterone concentrations throughout the menstrual cycle in women with PMS $(P>0.05)$. Plasma concentrations of oestradiol in patients with PMS were in the conventional normal range in both phases of the menstrual cycle, but were still significantly greater than those in controls in the luteal phase $(P<0.05)$. Allopregnanolone and progesterone responses to the GnRH injection are illustrated in Figs 1 and 2, and are represented as area under the curve (AUC) in Fig. 3. Although AUC is subject to increased error, as it represents the global response to the GnRH stimulation test, patients with PMS clearly had significantly lower concentrations of both progesterone and allopregnanolone with respect to symptom-free women $(P<0.01)$.

\section{Discussion}

The present study verified the presence of abnormal plasma concentrations of the neuroactive steroid, allopregnanolone, in patients suffering from PMS. PMS is a well acknowledged clinical disorder that has 
Table 1 Mean \pm S.E.M. basal concentrations of allopregnanolone and progesterone in the follicular phase and the luteal phase over 3 months.

\begin{tabular}{|c|c|c|c|c|}
\hline & \multicolumn{2}{|c|}{ Follicular phase } & \multicolumn{2}{|c|}{ Luteal phase } \\
\hline & Controls & PMS & Controls & PMS \\
\hline \multicolumn{5}{|c|}{ Allopregnanolone $(\mathrm{nmol} / \mathrm{l})$} \\
\hline First month & $0.81 \pm 0.05$ & $0.85 \pm 0.16$ & $3.89 \pm 0.14$ & $1.67 \pm 0.26^{\star \star \star}$ \\
\hline Second month & $0.77 \pm 0.04$ & $0.86 \pm 0.12$ & $3.83 \pm 0.09$ & $1.74 \pm 0.37^{* * *}$ \\
\hline Third month & $0.79 \pm 0.04$ & $0.88 \pm 0.10$ & $3.76 \pm 0.09$ & $1.54 \pm 0.20^{* * *}$ \\
\hline \multicolumn{5}{|c|}{ Progesterone $(\mathrm{nmol} / \mathrm{l})$} \\
\hline First month & $5.49 \pm 0.34$ & $2.43 \pm 0.27^{\star \star \star}$ & $36.03 \pm 0.84$ & $6.82 \pm 1.34^{\star \star *}$ \\
\hline Second month & $5.35 \pm 0.31$ & $2.47 \pm 0.29^{\star * *}$ & $34.89 \pm 0.76$ & $6.57 \pm 1.18^{\star * *}$ \\
\hline Third month & $5.22 \pm 0.25$ & $2.52 \pm 0.30^{\star * \star}$ & $35.10 \pm 0.78$ & $6.70 \pm 1.20^{\star * *}$ \\
\hline \multicolumn{5}{|l|}{ Oestradiol (pmol/l) } \\
\hline First month & $241.66 \pm 21.03$ & $239.89 \pm 24.55$ & $478.35 \pm 29.98$ & $585.67 \pm 29.87^{*}$ \\
\hline Second month & $249.02 \pm 23.89$ & $254.79 \pm 30.27$ & $469.74 \pm 31.19$ & $591.99 \pm 27.79^{*}$ \\
\hline Third month & $237.73 \pm 19.97$ & $247.79 \pm 27.49$ & $487.43 \pm 28.19$ & $573.99 \pm 31.09^{*}$ \\
\hline
\end{tabular}

* Significantly different with respect to controls: ${ }^{*} P<0.05$; ${ }^{* * \star} P<0.0001$.

been attributed to various hormonal abnormalities in the past. Recently, interest has turned to allopregnanolone as a possible causative factor of PMS. Although the initial studies showed no variations of serum concentrations of allopregnanolone in the luteal phase of patients with PMS compared with $(8,9)$, one of the studies demonstrated that greater luteal phase concentrations of allopregnanolone were associated with improved symptom ratings in patients with PMS (8). One study group found low concentrations of both progesterone and allopregnanolone in patients with PMS in the follicular phase only (10). Rapkin et al. (11) reported that women with PMS had lower luteal phase circulating concentrations of allopregnanolone, with no significant variations during the luteal phase (early mid- to late luteal phase) and normal concentrations of circulating progesterone (11). Our data clearly demonstrate the low concentrations of allopregnanolone in the luteal phase of women with PMS compared with those in controls. Moreover, the ratio between luteal phase and follicular phase concentrations of allopregnanolone in our patients with PMS was three times less than that of symptom-free women. Interestingly, progesterone plasma concentrations were also significantly lower in patients with PMS than in healthy individuals. Finally, whereas in normal women there is a positive correlation between allopregnanolone and progesterone concentrations throughout the menstrual cycle (12), this was not the case in our patients with PMS.

The reason why some women may have allopregnanolone deficiency remains unexplained. Our data are indicative of an impaired synthesis of this hormone by the corpus luteum or other steroidogenic organs (adrenals, liver, and adipose tissue). In fact, not only are the concentrations of its precursor, progesterone, reduced. We also found that bolus injection of GnRH during the luteal phase prompted a reduced allopregnanolone and progesterone response in women with PMS; this suggests that some sort of deficiency in corpus

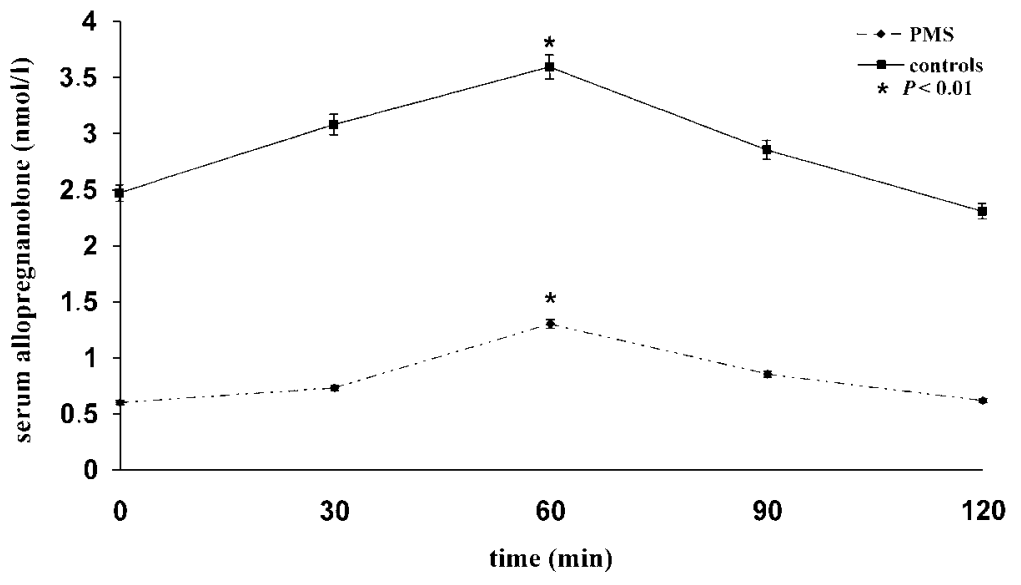

Figure 1 Time course of changes in allopregnanolone concentration during the $\mathrm{GnRH}$ test. 


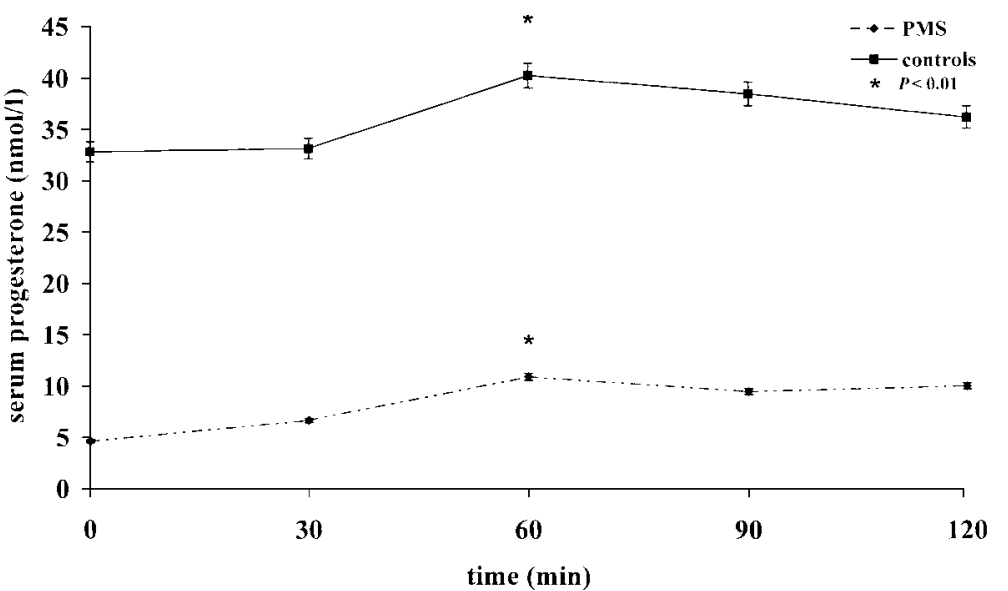

Figure 2 Time course of changes in progesterone concentration during the $\mathrm{GnRH}$ test. luteum function and synthesis of allopregnanolone and progesterone may underlie PMS. Women with PMS may have an inadequate luteal phase as a result of the underdevelopment of the large luteal cells of the granulosa, which are responsible for producing the greater part of progesterone and probably even allopregnanolone. Conversely, oestradiol concentrations are normal, and this is an indication that the small luteal cell apparatus is intact. Our patients with PMS had low basal concentrations of progesterone, but normal oestradiol concentrations. A large luteal cell deficiency (13) may be an intrinsic characterisic of such women, and this may explain why they experience symptoms of this nature over years, with a monthly recurrence. The low basal concentrations of allopregnanolone and progesterone, its precursor, observed in our patients with PMS and their abnormal response to GnRH suggest an impaired 'non-genomic' GABAmediated anxiolytic effect, and thus reduced sense of well-being during the luteal phase.

One must also take into account that, although allopregnanolone and progesterone concentrations are reduced in patients with PMS during the luteal phase, they are nevertheless greater than those in normal controls in the follicular phase. Therefore, the absolute concentrations of these steroids are probably not the only factors involved in producing PMS symptomatology. Further studies are needed at the receptor level in order to gain understanding of why some women face the 'blues' every month.
Recent reports support the notion that the biological key to the initiation of PMS may also lie in the altered 'genomic' effects of allopregnanolone at the central level. Smith et al. (7) demonstrated that, in rats, anxiety and seizure susceptibility are associated with sharp declines in circulating concentrations of allopregnanolone in the brain. They found that progesterone withdrawal altered GABA subunit activity, and that these effects were attributable to reduced concentrations of allopregnanolone. This neuroactive steroid enhances transcription of the gene encoding the $\alpha 4$ subunit of the $\mathrm{GABA}_{\mathrm{A}}$ receptor in the brain. These authors advanced the hypothesis that PMS symptoms may partly result from alterations in expression of $\mathrm{GABA}_{\mathrm{A}}$ receptor subunits as a result of progesterone withdrawal, and suggested that their animal model would be a useful aid to the understanding of PMS and post-partum syndrome. Premenstrual complaints may also be associated with decreased $\mathrm{GABA}_{\mathrm{A}}$ receptor sensitivity to pregnanolone in critical central nervous system sites, as areas controlling saccadic eye movements in patients with PMS in the late luteal phase are not affected by the administration of pregnanolone (14).

In conclusion, our data support the hypothesis that, although plasma concentrations of neuroactive steroids do not necessarily reflect those present at the central level, one cannot exclude the possibility that defective synthesis of a GABA-agonistic steroid may be responsible for the psychoneuroendocrine aspects of PMS.
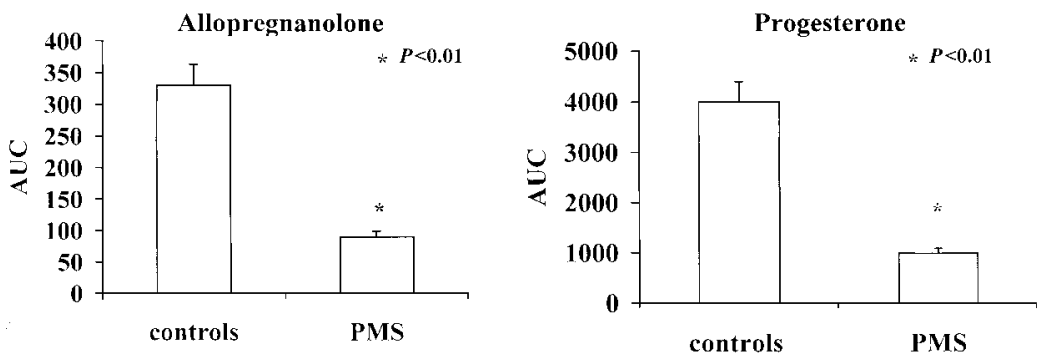

Figure 3 Response of allopregnanolone and progesterone to $\mathrm{GnRH}$, represented as AUC. 


\section{References}

1 Rubinow DR, Hoban MC, Grover GN, Galloway S, Roy-Byrne P, Andersen $\mathrm{R}$ et al. Changes in plasma hormones across the menstrual cycle in patients with menstrually related mood disorder and in control subjects. American Journal of Obstetrics and Gynecology 1988158 5-11.

2 Backstrom T, Sanders D, Leask R, Davidson D, Warner P \& Bancroft J. Mood, sexuality, hormones, and the menstrual cycle: hormone levels and their relationship to the Premenstrual syndrome. Psychosomatic Medicine 198345 503-507.

3 Seippel L \& Backstrom T. Luteal-phase estradiol relates to symptom severity in patients with premenstrual syndrome. Journal of Clinical Endocrinology and Metabolism 199883 19921998.

4 Schmidt PJ, Nieman LK, Danaceau MA, Adams LF \& Rubinow DR. Differential behavioural effects of gonadal steroids in women with and in those without premenstrual syndrome. New England Journal of Medicine 1998338 209-216.

5 Majewska MD, Harrison R \& Schwartz R. Steroid hormone metabolites are barbiturate-like modulators of the GABA receptor. Science 19862321004.

6 Majewska MD. Neurosteroids: endogenous bimodal modulators of the GABA-A receptor. Mechanism of action and physiological significance. Progress in Neurobiology 199238 379-395.

7 Smith SS, Gong QH, Hsu F-C, Markowitz RS, Ffrench-Mullen JM \& Li $\mathrm{X}$. $\mathrm{GABA}_{\mathrm{A}}$ receptor $\alpha 4$ subunit suppression prevents withdrawa properties of an endogenous steroid. Nature 1998392 926-930.

8 Wang M, Seippel L, Purdy RH \& Backstrom T. Relationship between symptom severity and steroid variation in women with premenstrual syndrome: study on serum pregnenolone sulfate,

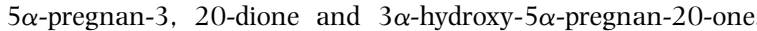

Journal of Clinical Endocrinology and Metabolism 199681 10761082.

9 Schmidt PJ, Purdy RH, Moore PH, Paul SM \& Rubinow DR. Circulating levels of anxiolytic steroids in the luteal phase in women with premenstrual syndrome and in control subjects. Journal of Clinical Endocrinology and Metabolism 199479 $1256-1260$.

10 Bicikova M, Dibbelt L, Hill M, Hampl R \& Starka L. Allopregnanolone in women with premenstrual syndrome. Hormone and Metabolic Research 199830 227-230.

11 Rapkin AJ, Morgan M, Goldman L, Brann DW, Simone D \& Mahesh VB. Progesterone metabolite allopregnanolone in women with premenstrual syndrome. Obstetrics and Gynecology 199790 709-714.

12 Genazzani AR, Petraglia F, Bernardi F, Casarosa E, Salvestroni C, Tonetti A et al. Circulating levels of allopregnanolone in humans: gender, age and endocrine influences. Journal of Clinical Endocrinology and Metabolism 199883 2099-2103.

13 Hinney B, Henze C, Kuhn W \& Wuttke W. The corpus luteum deficiency: a multifactorial disease. Journal of Clinical Endocrinology Metabolism 199681 365-370.

14 Sundstrom I, Andersson A, Nyberg S, Ashbrook D, Purdy RH \& Backstrom T. Patients with premenstrual syndrome have a different sensitivity to a neuroactive steroid during the menstrual cycle compared with control subjects. Neuroendocrinology $19987126-138$

Received 20 May 1999

Accepted 4 November 1999 